EGU2020-9270

https://doi.org/10.5194/egusphere-egu2020-9270

EGU General Assembly 2020

(c) Author(s) 2020. This work is distributed under

the Creative Commons Attribution 4.0 License.

\title{
Causal Discovery for Climate Time Series in the Presence of Unobserved Variables
}

\author{
Andreas Gerhardus and Jakob Runge \\ German Aerospace Center (DLR), Institute of Data Science, Jena, Germany
}

Scientific inquiry seeks to understand natural phenomena by understanding their underlying processes, i.e., by identifying cause and effect. In addition to mere scientific curiosity, an understanding of cause and effect relationships is necessary to predict the effect of changing dynamical regimes and for the attribution of extreme events to potential causes. It is thus an important question to ask how, in cases where controlled experiments are not feasible, causation can still be inferred from the statistical dependencies in observed time series.

A central obstacle for such an inference is the potential existence of unobserved causally relevant variables. Arguably, this is more likely to be the case than not, for example unmeasured deep oceanic variables in atmospheric processes. Unobserved variables can act as confounders (meaning they are a common cause of two or more observed variables) and thus introduce spurious, i.e., non-causal dependencies. Despite these complications, the last three decades have seen the development of so-called causal discovery algorithms (an example being FCl by Spirtes et al., 1999) that are often able to identify spurious associations and to distinguish them from genuine causation. This opens the possibility for a data-driven approach to infer cause and effect relationships among climate variables, thereby contributing to a better understanding of Earth's complex climate system.

These methods are, however, not yet well adapted to some specific challenges that climate time series often come with, e.g. strong autocorrelation, time lags and nonlinearities. To close this methodological gap, we generalize the ideas of the recent PCMCl causal discovery algorithm (Runge et al., 2019) to time series where unobserved causally relevant variables may exist (in contrast, $\mathrm{PCMCl}$ made the assumption of no confounding). Further, we present preliminary applications to modes of climate variability. 
Causal Discovery for Climate Time Series in the Presence of Unobserved Variables

Andreas Gerhardus and Jakob Runge

German Aerospace Center, Institute of Data Science, Jena

EGU2020: Sharing Geoscience Online

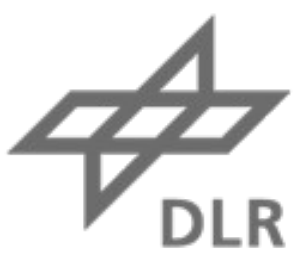




\section{Causal Relationships and Their Inference by Experimentation}

- Bypassing a major philosophical debate, we adopt the following definition of causality:

$X$ is a cause $Y$ if changing the value of $X$ while keeping all other conditions the same leads to a different value of $Y$

- The classical method of empirically inferring causal relationships is by experimentation:

Set up an experiment that changes the value of $X$ without affecting other variables. If the value of $Y$ changes when $X$ changes, then $X$ is a cause of $Y$

- Example:

$X$ : Ceiling light is on $\quad Y$ : Room is illuminated

$X$ is a cause of $Y$ : Turning the light on, the room is illuminated

$Y$ is not a cause of $X$ : Illuminating the room by, say, a flashlight does not turn on the light 


\section{Inference of Causal Relationships from Observational Data?}

- Causal discovery aims to infer causal relationships from observational data ${ }^{1}$

- Given the above definition of causality, this task comes with the following fundamental challenge:

The data is already there, it has been generated without us controlling the experimental conditions. That is, we cannot intervene to change the value of some variables and then observe what happens to the other variables

- How about statistical measures such as correlation or non-linear generalizations thereof, e.g. mutual information?

By themselves, statistical dependencies do not imply causation

- Then, is causal discovery possible at all?

Yes... ... when making some additional assumptions 


\section{A Framework for Causal Discovery}

- A common framework representing causal relationships is that of causal graphs and structural causal models (SCMs) ${ }^{1}$

- Causal graphs:

Nodes represent variables, arrows represent direct causal relationships

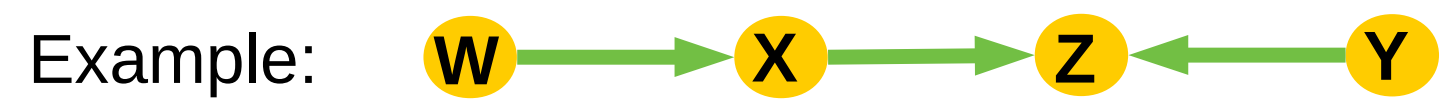

$\rightarrow X$ and $Y$ are direct causes of $Z$

$\rightarrow W$ is a direct cause of $X$ and an indirect cause of $Z$

- Structural causal models (which imply causal graphs):

Specification of the functional relationships that determine the value of each variable from those of the other variables

Example cont:: $X=f(W), \quad Z=g(X, Y)$

'See for example:

Pearl, J. Causality: Models, Reasoning, and Inference

Spirtes, P., Glymour, C., and Scheines, R. Causation, Prediction, and Search. Bollen, K. Structural Equations with Latent Variables. 


\section{Causal Graphs and Statistical Independencies: Part 1}

\section{- Assumption 1:}

The observed data was generated by a process that is expressable as SCM

- Discussion:

Equilibrium states of ordinary differential equations and random differential equations can be described by SCMs ${ }^{12}$

- Consequence:

The structure of the corresponding causal graph implies statistical independencies

Example cont: $\mathbf{W} \longrightarrow \mathbf{X} \longrightarrow \mathbf{Z} \longrightarrow-\mathbf{Y}$

$\Rightarrow W$ conditionally independent of $Z$ given $X \quad$ (causal influence is mediated by $X$ )

$\longrightarrow X$ and $W$ are marginally independent of $Y \quad$ (colliding arrows at $Z$ block influence)

General rule: d-separation ${ }^{3}$ 


\section{Causal Graphs and Statistical Independencies: Part 2}

- Assumption 2:

All statistical independencies are implied by d-separation on the causal graph ${ }^{1}$

- Discussion:

Intuitively, this excludes „accidental“ independencies due to fine-tuned parameters

Weaker forms of this assumption exist ${ }^{2}$

- Consequence:

Statistical independencies constrain the structure of the causal graph

- Constraint-based causal discovery:

Perform tests of statistical (in-)dependence in the observed data to constrain the causal graph as much as possible, thereby inferring causal relationships 


\section{Unobserved Causally Relevant Variables}

- In practice, we won't observe every single variable that is involved in the physical process under investigation

- However, some of the unobserved variables may be causally relevant:

$Z$ is causally relevant if it is a cause of two observed variables $X$ and $Y$ (and the causal influence of $Z$ on $Y$ is not entirely mediated through $X$, nor vice versa) ${ }^{1}$

If $Z$ is unobserved, it is called a hidden confounder or a hidden common cause

- This complicates the inference of causal relationships for the following reason:

Say we observe a statistical dependence between $X$ and $Y$, and this dependence cannot be blocked off by conditioning on some other observed variables

If there are no hidden confounders, we can conclude that $X$ causes $Y$ or vice versa

If there are hidden confounders, we cannot draw this conclusion 


\section{Causal Discovery With and Without Causal Sufficiency}

- Optional assumption: Causal Sufficiency

There are no hidden confounders, i.e., all causally relevant variables are observed

- Two important examples of constraint-based causal discovery algorithms:

- PC-Algorithm: Assumes causal sufficiency ${ }^{12}$

- FCl-Algorithm: Does not assume causal sufficiency 234

- Comparison:

- $\mathrm{FCl}$ makes fewer assumption as PC

- $\mathrm{FCl}$ is computationally and statistically more involved, it tends to assert fewer causal relationsships 


\section{Example of Discovering Causal Graphs with PC and FCI}

- Ground Truth:

$$
\mathbf{W} \longrightarrow \mathbf{X} \longrightarrow \mathbf{Z} \longrightarrow \mathbf{Y}
$$

$\Longrightarrow W$ conditionally independent of $Z$ given $W$

$\longrightarrow X$ and $W$ are marginally independent of $Y$

- Output of PC-Algorithm: 2 structures consistent with this exact set of independencies

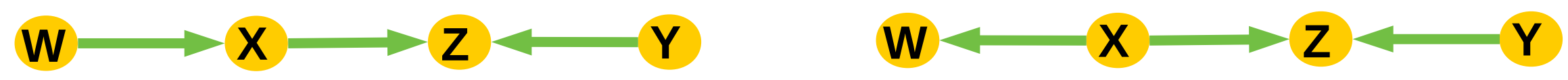

- Output of $\mathrm{FCl}$-Algorithm: 10 structures consistent with this exact set of independencies
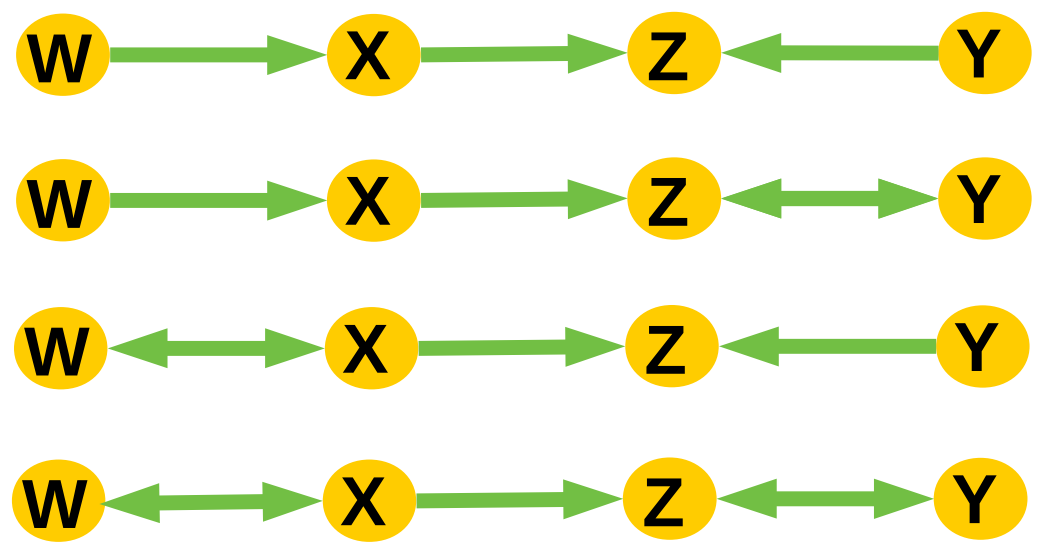
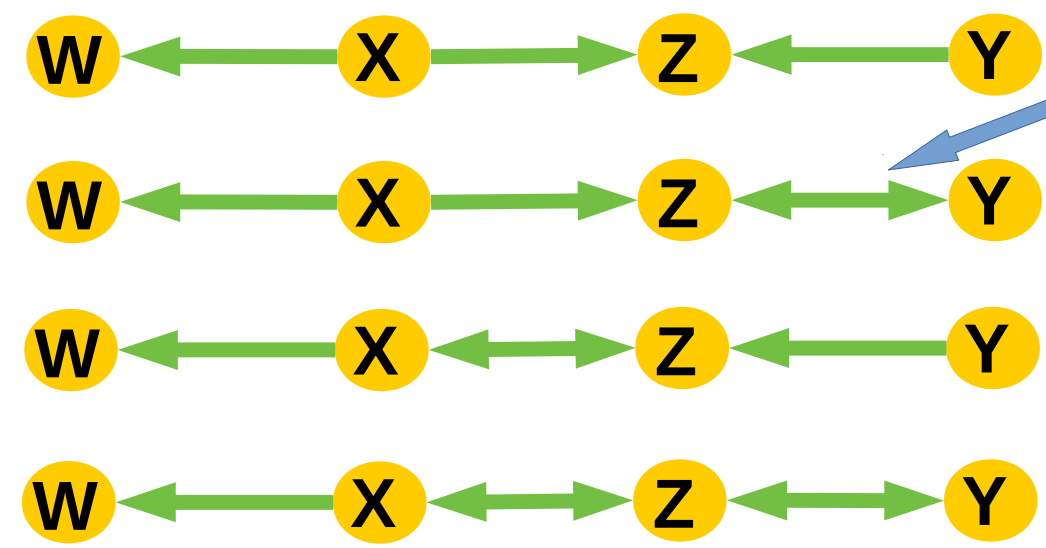

$=Z$ and $Y$ share hidden common cause 


\section{Challenges for Causal Discovery in Climate Time Series}

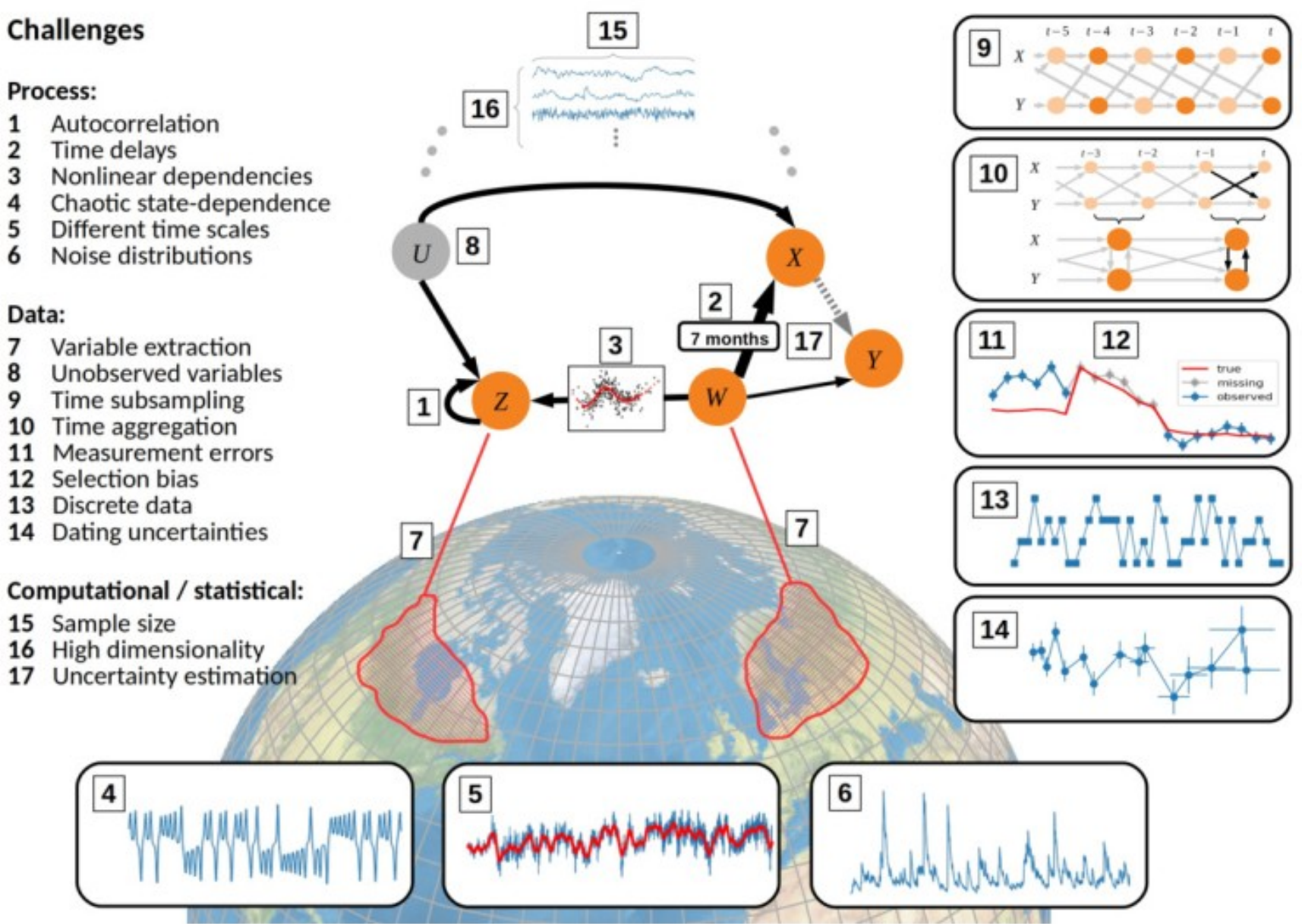

Picture from: Runge, J., Bathiany, S., Bollt, E. et al. Inferring causation from time series in Earth system sciences.
- Focus here:

\section{Autocorrelation}

- Challenge posed:

High rate of wrong statistical decisions

- Goal:

Modify and adapt causal discovery algorithms to become statistically more reliable and informative on autocorrelated climate time series data 


\section{Previous Work: Causal Discovery in Climate Time Series With Causal Sufficiency}

- In previous work we introduced the PCMCI-Algorithm, a modification of the PC-Algorithm to better handle autocorrelated time series ${ }^{1}$

- Central ideas of PCMCl:

- $\mathrm{MCl}$ conditional independence tests for well calibrated tests with improved detection power ${ }^{1}$

- Make fewer tests of statistical (in-)dependencies in total

- Result:

Significantly improved recall and well controlled false positives

- Limitation of PCMCl:

$\mathrm{PCMCl}$ makes the assumption of causal sufficiency, i.e., it assumes that there are no hidden confounders 


\section{Current Work: Causal Discovery in Climate Time Series Without Causal}

Sufficiency

- Currently, we are working on an algorithm that generalizes the key $\mathrm{PCMCl}$ ideas to the $\mathrm{FCl}-$ Algorithm, i.e., the case when there may be hidden confounders

- This requires significant changes and new conceptual ideas

- Numerical experiments are under way

- Outlook:

- Allow for selection bias

- Approach other challenges for causal discovery in climate time series ${ }^{1}$ 


\section{References}

- Pearl, J. Causality: Models, Reasoning, and Inference. Cambridge University Press, New York, USA, 2nd edition (2009)

- Spirtes, P., Glymour, C., and Scheines, R. Causation, Prediction, and Search. The MIT Press, Cambridge, Massachusetts, 2nd edition (2000).

- Peters, J., Janzing, D., and Schölkopf, B. Elements of Causal Inference: Foundations and Learning Algorithms. The MIT Press, Cambridge, Massachusetts (2017).

- $\quad$ Bollen, K. Structural Equations with Latent Variables. John Wiley \& Sons, New York, USA (1989).

- Mooij, J. M., Janzing, D., and Schölkopf, B. From Ordinary Differential Equations to Structural Causal Models: The Deterministic Case. UAl'13: Proceedings of the Twenty-Ninth Conference on Uncertainty in Artificial Intelligence (2013)

- Bongers, S., Mooij, J. M.. From Random Differential Equations to Structural Causal Models: The Stochastic Case. arXiv preprint arXiv:1803.08784 (2018).

- Verma, T. S., Pearl, J. Causal Networks: Semantics and Expressiveness. Machine Intelligence and Pattern Recognition vol. 9 (1990).

- Ramsey, J., Spirtes, P., and Zhang, J. Adjacency-Faithfulness and Conservative Causal Inference. UAl'06: Proceedings of the Twenty-Second Conference on Uncertainty in Artificial Intelligence (2006)

- Spirtes, P., Glymour, C. N. An algorithm for fast recovery of spares causal graphs. Social Science Computer Review vol. 9, issue 1 (1991)

- Spirtes, P., Meek, C., and Richardson, T. S. An algorithm for causal inference in the presence of latent variables and selection bias. UAl'95: Proceedings of the Eleventh conference on Uncertainty in artificial intelligence (1995)

- Zhang, J. On the completeness of orientation rules for causal discovery in the presence of latent confounders and selection bias. Artificial Intelligence vol. 172, issues 16-17 (2008)

- Runge, J., Bathiany, S., Bollt, E. et al. Inferring causation from time series in Earth system sciences. Nat Commun 10, 2553 (2019).

- Runge, J., Nowack, P., Kretschmer, M. et al. Detecting and quantifying causal associations in large nonlinear time series datasets. Science Advances vol. 5, no. 11 (2019) 